\title{
Melanoidins from coffee and lipid peroxidation
}

\author{
Davide Tagliazucchi
}

Corresponding author. Dr Davide Tagliazucchi: Department of Life Sciences, University of Modena and Reggio Emilia, Via Amendola 2, 42100 Reggio Emilia, Italy

Telephone: +39-0522-522060; fax: +39-0522-522027 E-mail: davide.tagliazucchi@unimore.it

Departmental-Institutional contact: Ms Silvia Ceretti, Department of Life Sciences, University of Modena and Reggio Emilia, Via Amendola 2, 42100 Reggio Emilia, Italy

Telephone: +39-0522-522051; fax: +39-0522-522027 E-mail: silvia.ceretti@unimore.it

Abbreviated version of the title/ running title: Coffee melanoidins and lipid peroxidation

Word count (excluding facing page, abstract, and references):

4926 words,

1 table,

5 figures,

44 references 


\begin{abstract}
Dietary lipid oxidation products are thought to be an important risk factor in the development of cardiovascular diseases. Lipid oxidation products may be already present in oxidized food and they may be generated during digestion of highly oxidizable food such as meat. In vitro and in vivo studies are consistent for a role of coffee high molecular weight melanoidins in the prevention of oxidative damage. Since coffee melanoidins are poorly bioavailable, the gastro-intestinal tract itself might constitute their main biological site of action where they reach high concentrations following coffee consumption. In the stomach they inhibit the peroxidation of meat lipids, decreasing the synthesis of hydroperoxides and secondary lipoxidation products. The reduction in the formation of these pro-atherogenic compounds has been shown to be followed by a decrease in their absorption in the human volunteers. The ability of coffee melanoidins to inhibit lipid peroxidation may contribute to their health benefits, since dietary oxidized lipid and advanced lipid oxidation endproducts are involved in the development of atherosclerosis and other diseases.
\end{abstract}

Key Words. Coffee melanoidins, lipid peroxidation, stomach, lipid hydroperoxide, antioxidants, meat, advanced lipid oxidation endproducts, diet, cardiovascular diseases. 
List of abbreviations.

ROS, reactive oxygen species;

ALEs, advanced lipid oxidation endproducts;

MDA, malondialdehyde;

4-HNE, 4-hydroxy-2-nonenal;

LDL, low-density lipoprotein;

LOOH, lipid hydroperoxide;

ABTS, 2,2'-azinobis-(3-ethylbenzothiazoline-6-sulfonic acid) radical cation;

DPPH, 2,2-diphenyl-1-picrylhydrazyl radical;

AAPH, 2,2'-azinobis-(2-amidinopropane)-dihydrochloride;

LH, linoleic acid;

$\mathbf{L} \cdot$, lipid radical;

LOO·, alkylperoxyl radical;

TBA-RS, thiobarbituric reactive substance;

$t$-BOOH, tert-butylhydroperoxide;

MRPs; Maillard reaction products. 


\section{INTRODUCTION}

Increasing evidence supports the hypothesis that lipid oxidative stress, lipid peroxidation and oxidative damage in the human body play a major role in developing most oxidative stress-related diseases.

Oxidative stress may be defined as an imbalance between the production of oxidants and the capacity of endogenous antioxidants systems to prevent oxidative damage that occur in the cell. Reactive oxygen species (ROS) such as superoxide anion and hydroxyl radical are among the most important oxidants generated during the oxidative stress condition. These free radicals can affect cell homeostasis causing damage to proteins, DNA and lipids Lipids (cholesterol and polyunsaturated fatty acids) are particularly vulnerable to oxidation initiated by free radicals. Lipid oxidation progress through an autocatalytic process that leads to the production and the accumulation of lipid oxidation products, in particular oxysterols and hydroperoxides. The latter can subsequently undergo fragmentation, generating a variety of highly reactive aldehydes, such as 2-alkenals (acrolein), 4-hydroxy-2-alkenals (4-hydroxy-2-nonenal, 4HNE) and ketoaldehydes (including malondialdehyde (MDA) and glyoxal), collectively known as advanced lipid oxidation endproducts (ALEs). Some of these products can spread easily and amplify the damage to DNA and protein initiated by ROS.

\section{LIPID PEROXIDATION AND DISEASES}

Lipid oxidation and ALEs have been linked to the pathogenesis of several diseases ${ }^{[1,2]}$. Lipid peroxidation occurs in all human neurodegenerative diseases. Lipid peroxidation products including free 4-HNE, acrolein, isoprostanes as well as 4-HNE and acrolein protein adducts are elevated in Alzheimer's diseased brains ${ }^{[3]}$. 4-HNE covalently modifies amyloid $\beta$ peptide via 1,4 -conjugate addition, and can crosslink amyloid $\beta$ peptides to each other, triggering its aggregation and 
accelerating the formation of protofibrils ${ }^{[4]}$. Both 4-HNE and acrolein are neurotoxic in a time- and concentration-dependent manner for hippocampal cultured cells ${ }^{[1]}$.

Elevated levels of 4-HNE protein adduct were also found in subjects with Parkinson disease and amyotrophic lateral sclerosis ${ }^{[1]}$.

Oxidative stress and lipid oxidation play also an important role in the pathogenesis of atherosclerosis. LDL oxidation leads to the formation of peroxidized lipids which can breakdown into reactive aldehyde that are responsible for the oxidative damage to the LDL apoprotein ${ }^{[5]}$. Oxidized phospholipids are present in oxidized LDL, accumulate in atherosclerotic lesions and are able to trigger vascular inflammation and pro-atherogenic reactions ${ }^{[6]}$. 4-HNE- and MDA-adducts are detected in atherosclerotic lesions ${ }^{[1,2]}$. ALEs react with apoprotein $\mathrm{B}$, altering its structure and increasing its affinity for the scavenger receptors in macrophages leading to massive cholesterol accumulation ${ }^{[7]}$. There is also evidence that oxycholesterols are angiotoxic, pro-inflammatory and pro-atherogenic ${ }^{[8]}$.

It is still unclear how and when LDL are oxidized. It has been suggested that LDL may be oxidized directly in the sub-intima space of the arterial wall ${ }^{[9]}$ and/or oxidized in other sites and transferred from plasmatic circulation to the atherosclerotic lesions ${ }^{[10]}$. The pro-atherogenic oxidized LDL in the circulation are partially derived from oxidized lipids in the diet ${ }^{[11]}$. Oxidized fatty acids and oxidized cholesterols in the diet are absorbed in the small intestine and transferred into the chylomicrons ${ }^{[12,13]}$. Oxidized chylomicrons are not considered potentially atherogenic, but the oxidized remnants can increase the atherogenic risk by depositing itself in the vessel wall and increasing the oxidative stress ${ }^{[14]}$. While the oxidized fatty acids are not transferred to other lipoproteins, oxidized cholesterol is directly transferred from chylomicrons to LDL in the circulation contributing, at least partially, to the formation of oxidized LDL ${ }^{[12,13]}$. 
Also dietary ALEs, such as MDA, can be absorbed and accumulated in human plasma after meat consumption ${ }^{[15]}$. MDA from the diet causes oxidative LDL post-prandial modification in humans $[16]$.

\section{SOURCES OF DIETARY LIPID OXIDATION PRODUCTS AND ADVANCED LIPID OXIDATION ENDPRODUCTS}

The first source of dietary oxidized lipid and ALEs is represented by the oxidative reaction that occurs during processing, storage and cooking of food, especially in meat products.

In food, free ferrous iron is a primary catalyst for the initiation of lipid peroxidation because of its ability to generate hydroxyl radical from oxygen. Low concentrations of reducing agents, such as ascorbic acid, drives the reduction of ferric to ferrous iron promoting lipid peroxidation in muscle tissue. On the other hand, heme-containing proteins (such as myoglobin and hemoglobin) as well as iron-oxygen complexes (ferryl and oxo-ferryl radicals) are also considered as initiators of lipid peroxidation in meat and meat products ${ }^{[17]}$.

The primary compounds formed during lipid oxidation are lipid hydroperoxides (LOOH) whose concentration can reach $200 \mu \mathrm{M}$ in cooked meat ${ }^{[18]}$. The level of lipid hydroperoxides greatly increases after cooking. Kanner and Lapidot ${ }^{[18]}$ found that the level of lipid hydroperoxides in cooked red turkey meat was about 100-fold higher than the value found in raw red turkey meat. The $\mathrm{LOOH}$ and lipid free radicals generated during the oxidative reaction produce ALEs (MDA, acrolein, and 4-HNE) and are able to co-oxidize a variety of vitamins (A, E, and C) and cholesterol

${ }^{[19]}$. The concentration of ALEs in meat and fish products could exceed $300 \mu \mathrm{M}^{[20]}$.

The amount of oxidized cholesterol increases during cooking, depending on the type and time of cooking, and frozen storage in meat products and can reach concentrations of 10 to $100 \mu \mathrm{M}^{[20]}$. The second source of dietary oxidized lipid and ALEs is the stomach. The idea of the stomach as a "bioreactor" in which the lipid oxidation is enhanced, was first proposed by Kanner and co-workers 
${ }^{[18,19]}$. The gastric fluid, which contains dissolved oxygen and has a low $\mathrm{pH}$, is an optimal medium for further dietary lipid oxidation in meat. The amount of LOOH in meat incubated in the gastric fluid for 3 hours at $\mathrm{pH} 3$ increased from 6 to 8 -fold, with a final concentration ranging from 500 to $1200 \mu \mathrm{M}$, depending on the types of meat ${ }^{[18,21,22]}$. ALEs are formed during the gastric digestion of meat. Their concentration, measured as MDA equivalent increase by more than 2-fold during meat incubation in gastric fluid reaching concentration of more than $100 \mu \mathrm{M}^{[16,21-23]}$.

Typically, lipid peroxidation in gastric fluid occurs in the presence of catalysts, such as free iron ions, free heme group or heme-containing peptides/proteins such as metmyoglobin. We studied the role of non-heme iron on gastric lipid peroxidation by incorporating EDTA in the simulated gastric digestion of turkey meat ${ }^{[21,22]}$. The maximum amount of inhibition in the formation of hydroperoxides in the gastric fluid was $68.7 \%$ at EDTA concentration of $0.4 \mathrm{mM}$. The increase in EDTA concentration did not cause a further increase in the inhibition. These results suggest that lipid peroxidation in turkey meat is affected mainly by non-heme iron. However, a significant part of lipid peroxidation seems to be independent of free iron ion and attributable to the catalytic role of heme iron.

Pepsin activity is important for gastric lipid peroxidation since the omission of pepsin from the simulated gastric fluid cause an inhibition of about 55\% in the level of hydroperoxides after 180 minutes of gastric digestion of turkey meat ${ }^{[21,22]}$. This "prooxidant effect" of pepsin may be due to the liberation from digested proteins of transition metals such as iron and copper that are initiators of lipid peroxidation. Moreover, an enhanced prooxidative activity of metmyoglobin was seen after pepsin hydrolysis, suggesting that myoglobin fragments with high prooxidative activity are formed under conditions of proteolysis ${ }^{[24]}$. 


\section{COFFEE MELANOIDINS: STRUCTURE AND BIOLOGICAL ACTIVITY}

Melanoidins are generically defined as brown-coloured, nitrogen-containing high molecular weight (usually more than $5 \mathrm{KDa}$ ) compounds formed in the last stage of the Maillard reaction. In coffee, melanoidins are formed during the roasting process and are responsible for the coffee colour. The mean melanoidins content in roasted coffee powder is $7.2 \%$ whereas in the coffee brew the melanoidins concentration ranges between 1.7 and $4.3 \mathrm{~g} / \mathrm{l}$ depending on the method of preparation ${ }^{[25]}$. Polysaccharides (mainly galactomannans and arabinogalactans), proteins, and chlorogenic acids, contribute to the formation of coffee melanoidins, although most of the weight of the coffee melanoidins (up to $90 \%$ ) is composed of unknown material ${ }^{[26]}$. Some low molecular weight compounds (chlorogenic acids and low molecular weight Maillard reaction products) have been found to be ionically linked to the melanoidin core ${ }^{[27]}$. Despite the many efforts made in recent years, the chemical structure of melanoidins is largely unknown and different melanoidin populations may coexist within the coffee with different abundances in the different melanoidin populations ${ }^{[33]}$. Due to the complexity of the melanoidins population in coffee, the approach for the isolation of coffee melanoidins take advantage of their molecular weight. The most utilized involve the use of dialysis tubing or ultrafiltration apparatus with a molecular weight cut-off of 5-10 KDa [26].

The estimated daily intake of coffee melanoidins ranges between 200 to $2600 \mathrm{mg}$ per capita per day with an estimated worldwide intake of about $900 \mathrm{mg}$ per capita per day ${ }^{[25]}$. The daily intake, however, could be much higher for heavy coffee drinkers ( $>6$ cups per day) which can easily reach more than $5 \mathrm{~g}$ of coffee melanoidins per day ${ }^{[25]}$.

Considering the high intake of coffee melanoidins, their biological activity and potential impact on human health is a topic of great interest. Different in vitro biological activities have been attributed to coffee melanoidins, namely, antioxidant, antimicrobial, anti-inflammatory, antiglycative and 
antihypertensive activities as well as the ability to inhibit matrix metalloproteases and to modulate the bacterial colonic population ${ }^{[26]}$.

Two major factors limit so far the actual physiological relevance of the biological activities of coffee melanoidins. First, the limited knowledge of the structure of the coffee melanoidins makes it difficult to identify the active principles responsible for the specific biological activity.

Most studies have been carried out using the high molecular weight material (usually higher than 10 $\mathrm{KDa})$ isolated from coffee without further purification. Secondly, although melanoidins are consumed regularly as part of the daily human diet, they are generally considered as poorly absorbable and bio-available compounds ${ }^{[28]}$. The absorption of the melanoidins is dependent of their molecular weight and solubility. The absorption of the low molecular weight and water soluble melanoidins seems to be favoured. In rats 70 to $90 \%$ of orally ingested high molecular weight melanoidins are excreted in the faeces, and only 1 to $5 \%$ absorbed and excreted in the urine ${ }^{[28]}$. Regarding coffee melanoidins, no study has been conducted in order to determine their bioavailability and metabolism in vivo and, so far, there is no evidence for the ability of coffee melanoidins to cross intact the intestinal mucosal barrier.

For the reasons above stated, it is unlikely that coffee melanoidins could act as biologically active compounds in the bloodstream or organs. More important, most of the consumed melanoidins remain in the gastrointestinal tract, therefore, it may be a key site for their antioxidant and biological action. In addition, coffee high molecular weight melanoidins seem not to be degraded in the first portion of the gastrointestinal tract ${ }^{[28,29]}$. In the last ten years some studies have suggested that they can play a relevant role in the gastrointestinal tract.

Coffee melanoidins act as prebiotic, promoting selective bacterial growth in the colon ${ }^{[30]}$. Also, they can have a role in the protection against cancer development in the colon by inhibiting matrix metalloproteases ${ }^{[31]}$. Indeed, the coffee melanoidins fermented by human faecal bacteria 
retain antioxidant activity ${ }^{[30]}$. The high molecular weight coffee melanoidins can also act as inhibitors of lipid peroxidation in the stomach, suggesting a role in the prevention of oxidative damage ${ }^{[21]}$.

The rest of this chapter focuses on the antioxidant activity of coffee melanoidin with special emphasis on the ability of these compounds to inhibit lipid peroxidation.

\section{COFFEE MELANOIDINS AS RADICAL SCAVENGERS, METAL CHELATORS AND LIPID PEROXIDATION INHIBITORS}

Several studies carried out in recent years have shown that melanoidins extracted from coffee possess antioxidant activity. The antioxidant activity of melanoidins isolated from the different brews was studied using different methodologies. The techniques most frequently used, measured the radical scavenging ability of coffee melanoidins against stable free radicals such as 2,2 'azinobis-(3-ethylbenzothiazoline-6-sulfonic acid) radical cation (ABTS) and 2,2-diphenyl-1picrylhydrazyl radical (DPPH). Delgado-Andrade et al. ${ }^{[32]}$ showed that the radical scavenging ability of coffee melanoidins isolated by ultrafiltration with a nominal cut-off of $10 \mathrm{KDa}$ from instant coffee was higher in the dark-roasted coffee using DPPH method. Data from the ABTS method showed no significant differences in radical scavenging ability of coffee melanoidins as a function of the degree of roasting. Also Del Pino-García ${ }^{[33]}$ et al. found no differences in the radical scavenging activity measured with the ABTS assay of instant coffee melanoidins (> $10 \mathrm{KDa})$ as a function of the degree of roasting. However, they found a decrease in the DPPH radical scavenging activity with the increasing degree of roasting. Conversely, Borrelli et al. ${ }^{[34]}$ showed that the ABTS radical scavenging activity of coffee melanoidins (isolated by gel filtration chromatography and with a molecular weight between 1.2 and $4.7 \mathrm{KDa}$ ) decreased as the roasting severity increased whereas no significant differences were observed with the DPPH assay. It is worth noting that 
comparisons between these studies should take into account differences in melanoidin isolation protocols and in coffee brew used (espresso, filter coffee or instant coffee).

The contribution of high molecular weight melanoidins to the radical scavenging activity of coffee brews range between 10 and $38 \%{ }^{[29,33,35]}$. The radical scavenging activity of coffee melanoidins is mainly due to the phenolic moieties incorporated and/or condensed in the melanoidin skeleton during the roasting process ${ }^{[35]}$. The hypothesis was reinforced by the fact that a coffee melanoidin fraction, obtained after hydrophobic chromatography of a $>3 \mathrm{KDa}$ fraction, rich in noncarbohydrate and non-protein chromophore and phenolic moieties showed three- to four-fold higher radical scavenging activity than the remaining high molecular-weight material ${ }^{[36]}$. In addition, low molecular weight compounds ionically linked to the melanoidin core (phenolic groups and/ or low molecular weight Maillard reaction products), released after incubation of high molecular weight melanoidins with a molecular mass more than $10 \mathrm{KDa}$ in $2 \mathrm{M} \mathrm{NaCl}$, exhibited higher radical scavenging activity than the remaining polymeric material ${ }^{[29,32]}$. Among the different foods, coffee melanoidins isolated by ultrafiltration (>10 KDa) exhibited sixfold higher radical scavenging activity than traditional balsamic vinegar melanoidins and eight- and eleven-fold higher radical scavenging activity than barley coffee and dark beer melanoidins, respectively ${ }^{[21,22]}$.

The radical scavenging activity of coffee melanoidins was also tested against the hydroxyl radical. In this regard, coffee melanoidins isolated by ultrafiltration $(>10 \mathrm{KDa})$ were more efficient among melanoidins from beers and sweet wine ${ }^{[37]}$. The hydroxyl radical scavenging ability of coffee melanoidins was not affected by the degree of roasting ${ }^{[33]}$. Antioxidant properties of coffee melanoidins can result from their free radical scavenging activity but their ability to chelate transition metal ions also play an important role. Coffee melanoidins are able to bind $\mathrm{Zn}^{2+}, \mathrm{Cu}^{2+}$, and $\mathrm{Fe}^{2+[26,38,39]}$. Most studies have focused on the ability of coffee melanoidins to chelate ferrous iron. Coffee melanoidins isolated by ultrafiltration (> $10 \mathrm{KDa}$ ) were 
more efficient iron-chelators than melanoidins from beers, sweet wine, barley coffee and traditional balsamic vinegar ${ }^{[21,22,39]}$.

Free radical scavenging activity and metal-chelating ability are key factors for the capacity to inhibit lipid peroxidation, therefore, it is not surprising that coffee melanoidins are efficient inhibitors of lipid peroxidation.

Table 1 represents a summary of the in vitro and in vivo studies involving coffee melanoidins as inhibitors of lipid peroxidation.

The anti-peroxidative activity of coffee melanoidins has been tested in different model systems. The inhibition of linoleic acid (LH) oxidation in aqueous system has been extensively used. In this system the peroxidation of LH is inititiated by a water-soluble molecules (2,2'-azinobis-(2amidinopropane)-dihydrochloride; AAPH) which produce a lipid radical (L·) which in the presence of oxygen is converted into an alkylperoxyl free radical (LOO·). The LOO free radical, subsequently, abstracts a hydrogen atom from LH (propagation step) generating a new LOO free radical and a stable $\mathrm{LOOH}$. This method is based on the rate of oxidation of $\mathrm{LH}$ to its conjugated diene hydroperoxide in aqueous media in the presence or absence of the antioxidant compound ${ }^{[40]}$. In this system the lipid peroxidation is independent of the presence of transition metals and, therefore, it is useful to study the chain-breaking activity of an antioxidant. Borrelli et al. ${ }^{[34]}$ showed that the ability of coffee melanoidins (isolated by gel filtration chromatography and with a molecular weight between 1.2 and 4.7 KDa) to prevent lipid peroxidation in LH system increased as the roasting severity increased. They also found that the high molecular fraction of green coffee brew was completely ineffective in inhibiting LH oxidation. In another study ${ }^{[27]}$, the anti-peroxidative activity of coffee melanoidins isolated by ultrafiltration $(>10 \mathrm{KDa}$ ) and tested against $\mathrm{LH}$ oxidation in an aqueous system was independent of the degree of roasting. Coffee melanoidins $(>10 \mathrm{KDa})$ show higher anti-peroxidative activity than melanoidins isolated from sweet wine and beers ${ }^{[40]}$. The anti-peroxyl radical activity of coffee melanoidins in 
LH systems is mainly due to the low molecular weight compounds ionically bound to the polymeric melanoidins ${ }^{[27]}$. In the proposed mechanism of action ${ }^{[40]}$, coffee melanoidins may react with LOO. free radical instead of $\mathrm{LH}$, reducing the propagation state of $\mathrm{LH}$ oxidation. Coffee melanoidins may act as chain-breaking antioxidants, transferring a hydrogen atom to the free radical LOO to produce the stable $\mathrm{LOOH}$ and a stable melanoidin radical (reaction 1).

$$
\mathrm{LOO}+\mathrm{M}-\mathrm{OH} \rightarrow \mathrm{LOOH}+\mathrm{M}-\mathrm{O}
$$

The anti-peroxyl radical activity of coffee melanoidins has also been evaluated ex vivo in a biological system using rat liver microsomal preparations. The extent of lipid peroxidation was assayed by measuring the thiobarbituric reactive substance (TBA-RS) after induction of oxidative damage with an inititiator. The most active coffee melanoidins, isolated by dialysis ( $>3.5 \mathrm{KDa})$ and subsequent gel filtration chromatography, were found to be the medium and, especially, the lower molecular weight melanoidins ${ }^{[41]}$. The inhibition of rat liver microsomal peroxidation decreased from light- to dark-roasted coffee ${ }^{[33]}$.

Lipid peroxidation inhibitory activity of coffee melanoidins isolated by ultrafiltration (> $10 \mathrm{KDa})$ and subjected to consecutive gastro-pancreatic digestion was assayed on human hepatoma HepG2 cells ${ }^{[42]}$. Coffee melanoidins showed protective effects on hepatoma cells submitted to oxidative stress induced by the prooxidant tert-butylhydroperoxide ( $t$-BOOH). Coffee melanoidins completely abolished the formation of TBA-RS in the cells already at a concentration of $0.5 \mu \mathrm{g} / \mathrm{mL}$. More interestingly, the pre-treatment of hepatoma cells with $5-10 \mu \mathrm{g} / \mathrm{mL}$ of digested coffee melanoidins completely avoided the $t$-BOOH-induced increase of TBARS. The cells were exposed to the digested coffee melanoidins for 2 hours, followed by washing, so that the extracellular presence of the coffee melanoidins was precluded when treatment with $t$-BOOH commenced. High 
molecular weight coffee melanoidins were found to be non-cytotoxic at concentrations upto 100 $\mu \mathrm{g} / \mathrm{mL}$.

Coffee melanoidins were also tested for their ability to inhibit copper induced oxidation of human $\mathrm{LDL}^{[43]}$. LDL oxidation was spectrophotometrically monitored by measuring the formation of conjugated diene at $234 \mathrm{~nm}$. Coffee melanoidins resulted more effective than other thermally processed food.

\section{COFFEE MELANOIDINS INHIBIT LIPID PEROXIDATION DURING SIMULATED DIGESTION OF MEAT}

The ability of melanoidins isolated by ultrafiltration ( $>10 \mathrm{KDa}$ ) from instant coffee to inhibit lipid peroxidation was also tested in an in vitro model of gut during digestion of meat ${ }^{[21]}$. In our initial experiments, we examined whether coffee melanoidins were able to inhibit the formation of $\mathrm{LOOH}$ during the in vitro gastric digestion of turkey meat. As shown in figure 1, coffee melanoidins were able to decrease the lipid peroxidation during the simulated gastric digestion of turkey meat and at a concentration of $3 \mathrm{mg} / \mathrm{mL}$ reversed the reaction and broke down hydroperoxides to a concentration lower than the initial value. The anti-peroxidative activity of high molecular weight coffee melanoidins is concentration-dependent. The time course of the peroxidation of turkey meat in the presence of coffee melanoidins showed that at both the tested concentrations, the inhibitory effect was already significant after 30 minutes of incubation.

We next examined the ability of coffee melanoidins to decrease the formation of ALEs measured as TBA-RS (figure 2). The inhibitory effect was significant from 30 minutes for $3 \mathrm{mg} / \mathrm{ml}$ and only at 150 and 180 minutes for $1.5 \mathrm{mg} / \mathrm{ml}$ of coffee melanoidins. When coffee melanoidins were coincubated in the gastric fluid with turkey meat at $3 \mathrm{mg} / \mathrm{ml}$ concentration we found an inhibition of $91 \%$ in the TBA-RS formation. 
To gain more information on the mechanism of action of coffee melanoidins we tested the ability of these compounds to scavenge free radicals and to chelate iron and heme, as well as their possible effect on pepsin activity.

Firstly, instant coffee melanoidins are effective in scavenging ABTS radical cation (with a TEAC value of $190 \mathrm{mg} / \mathrm{g}$ of melanoidins) through a hydrogen-atom transfer mechanism. Secondly, instant coffee melanoidins are able to chelate ferrous ions (figure 3) and heme (figure 4). The $K d$ value for $\mathrm{Fe}^{2+}$ binding was $3.8 \mathrm{mg} / \mathrm{ml}$ of melanoidin and the Hill coefficient was 2.6 suggesting the presence of multiple $\mathrm{Fe}^{2+}$ binding sites in coffee melanoidins.

The binding of high molecular weight melanoidins with heme caused a reduction in the absorbance of the Soret band at $400 \mathrm{~nm}$ (figure 4). The calculated $K d$ value was $0.2 \mathrm{mg} / \mathrm{ml}$ of melanoidin showing that coffee melanoidins have a great affinity towards heme. The Hill coefficient higher than one (1.52) indicates a positive cooperativity in the binding suggesting the presence of more than one binding site for heme in food melanoidins.

Finally, coffee melanoidins were not able to inhibit pepsin activity.

On the basis of the above reported evidence, figure 5 shows the proposed mechanism of action for the anti-peroxidative activity of coffee melanoidins in the in vitro gastric system. As shown, instant coffee melanoidins may act at different levels. In the first step of the reaction (figure 5A) they chelate $\mathrm{Fe}^{2+}$ ions and heme preventing the formation of alkoxyl radical and of the protonated ferryl species $\left(\mathrm{HmFe}^{4+}-\mathrm{OH}\right)$ of heme. In the second step of the reaction, high molecular weight coffee melanoidins act as radical scavengers (figure 5B) by reacting directly with peroxyl radical preventing the propagation step.

It has been shown that the anti-peroxidative activity of coffee melanoidins is due to its high content in phenolic groups ${ }^{[21]}$. Coffee melanoidins were more efficient in inhibiting lipid peroxidation during the simulated digestion of turkey meat than melanoidins from dark beer, barley coffee and traditional balsamic vinegar ${ }^{[21,22]}$. 


\section{IN VIVO EVIDENCE OF COFFEE MELANOIDINS AS INHIBITORS OF LIPID PEROXIDATION}

The in vivo evidence of the anti-peroxyl radical activity of coffee melanoidins are scarce but increasing.

The first evidence that food-derived Maillard reaction products elicit anti-peroxidative properties in humans was reported by Dittrich et al. ${ }^{[43]}$. The authors delivered to eight human subjects a diet containing food rich in MRP. The first week of the diet was used as the wash-out period in which roasted, baked and fried products were not consumed. In the second week of the intervention study they administered to the subjects a diet rich in dark beer, bread crusts, and roasted coffee. It was shown that the MRP-rich diet led to a statistically significant protection of isolated LDL against copper-induced oxidation ex vivo. There are some limitations in the study. It is not possible to understand which or what foods are most effective in vivo and it is not possible to discriminate the possible effect of other components (such as polyphenols) present in the tested foods.

In a recent work, Kanner and co-worker ${ }^{[23]}$ administered to human volunteers a meal consisting of $250 \mathrm{gr}$ of fast-food meat and $200 \mathrm{~mL}$ of coffee beverage or water as control. The purpose of the study was to verify if the simultaneous consumption of coffee and meat led to a reduction in the absorption of ALEs. They measured the plasmatic level of MDA and found that the consumption of roasted coffee during a meal of fast-food meat, resulted after 2 and $4 \mathrm{~h}$, in the inhibition by $80 \%$ and $50 \%$, respectively, of postprandial plasma MDA absorption. Although it was not possible adequately to identify the molecules (polyphenols and/or melanoidins) responsible for the effect, in vitro data ${ }^{[27]}$ strongly support the idea that high molecular weight coffee melanoidins are mainly responsible for the anti-peroxidative effect of coffee found in vivo.

In another study by Vitaglione et al. ${ }^{[44]}$, coffee and its components (polyphenols and melanoidins extracted by dialysis $>12 \mathrm{KDa}$ ) were studied for their ability to reduce liver damage and systemic 
lipid peroxidation in rats. Rats were fed with a high-fat diet and different beverages, coffee or a solution of coffee polyphenols or a solution of coffee melanoidins. Coffee was able to reduce serum MDA concentration by $26 \%$ compared to un-treated rats. Regarding the effect of coffee components, although in the polyphenols-feeding rats a more pronounced effect on the serum levels of MDA was observed (-20\% compared to un-treated rats), even in melanoidins-feeding rats a slight reduction in plasma levels of MDA was observed (-10\% compared to un-treated rats).

\section{CONCLUSION}

In vitro and in vivo studies are consistent for a role of coffee high molecular weight melanoidins in the prevention of oxidative damage. Numerous studies reported the in vitro radical scavenging activity, chelating ability and antiperoxidative activity of coffee melanoidins. Due to their low bioavailability, it is unlikely that coffee melanoidins can exert their protective effects at the systemic level. More plausibly, coffee melanoidins can act at gastro-intestinal level where they reach high concentration following coffee consumption. In the stomach they act as antioxidants and metal chelators, inhibiting the peroxidation of meat lipids and decreasing the synthesis of hydroperoxides and secondary lipoxidation products. The reduction in the formation of these proatherogenic compounds has been shown to be followed by a decrease in their absorption in human volunteers. The ability of melanoidins to inhibit lipid peroxidation may contribute to their health benefits, since dietary oxidized lipid and ALEs are involved in the development of atherosclerosis and other diseases. 


\section{SUMMARY POINTS}

- In vitro and in vivo studies are consistent for a role of coffee melanoidins in the prevention of oxidative damage.

- Lipid peroxidation has been linked to oxidative stress-related diseases.

- Dietary lipid oxidation products are a source of oxidized lipoproteins in the circulation.

- Dietary lipid oxidation products are present in cooked meat and their concentration dramatically increase during gastric digestion.

- Numerous studies report the in vitro radical scavenging activity, chelating ability and antiperoxidative activity of coffee melanoidins.

- Coffee melanoidins decrease the synthesis of hydroperoxides and secondary lipoxidation products during in vitro gastric digestion of meat

- Coffee consumption with meat bring about a decrease in the absorption of secondary lipoxidation products in human volunteers. 


\section{REFERENCES}

1. Negre-Salvayre, A., Auge, N., Ayala, V., Basaga, H., Boada, J., Brenke, R., Chapple, S., Cohen, G., Feher, J., Grune, T., Lengyel, G., Mann, G. E., Pamplona, R., Poli, G., PorteroOtin, M., Riahi, Y., Salvayre, R., Sasson, S., Serrano, J., Shamni, O., Siems, W., Siow, R. C. M., Wiswedel, I., Zarkovic, K., and Zarkovic, N. (2010). Pathological aspects of lipid peroxidation. Free Rad Res 44, 1125-1171.

2. Uchida, K. (2000). Role of reactive aldehyde in cardiovascular diseases. Free Rad Biol Med $28,1685-1696$.

3. Butterfield, D. A., Bader Lange, M. L., and Sultana, R. (2010). Involvements of the lipid peroxidation product, HNE, in the pathogenesis and progression of Alzheimer's disease. Biochim Biophys Acta 1801, 924-929.

4. Siegel, S. J., Bieschke, J., Powers, E. T., and Kelly, J. W. (2007). The oxidative stress metabolite 4-hydroxynonenal promotes Alzheimer protofibril formation. Biochemistry 46, $1503-1510$.

5. Etserbauer, H, Wag, G., and Puhl, H. (1993). Lipid peroxidation and its role in atherosclerosis. Br Med Bull 49, 566-576.

6. Leitinger, N. (2005). Oxidized phospholipids as triggers of inflammation in atherosclerosis. Mol Nutr Food Res 49, 1063-1071.

7. Kreuzer, J., White, A. L., Knott, T. J., Jien, M. L., Mehrabian, M., Scott, J., Young, S. G., and Haberland, M. E. (1997). Amino terminus of apolipoprotein B suffices to produce recognition of malondialdehyde-modified low density lipoprotein by the scavenger receptor of human monocyte-macrophages. J Lipid Res 38, 324-342. 
8. Leonarduzzi, G., Sevanian, A., Sottero, B., Arkan, M. C., Biasi, F., Chiarpotto, E., Basaga, H., Poli, G. (2001). Up-regulation of the fibrogenic cytokine TGF-beta1 by oxysterols: a mechanistic link between cholesterol and atherosclerosis. FASEB J 15, 1619-1621.

9. Palinski, W., Rosenfeld, M. E., Ylä-Herttuala, S., Gurtner, G. C., Socher, S. S., Butler, S. W., Parthasarathy, S., Carew, T. E., Steinberg, D., and Witztum J. L. (1989). Low density lipoprotein undergoes oxidative modification in vivo. PNAS 86, 1372-1376.

10. Tsimikas, S., Aikawa, M., Miller Jr, F. J., Miller, E. R., Torzewski, M., Lentz, S. R., Bergmark, C., Heistad, D. D., Libby, P., and Witztum, J. L. (2007). Increased plasma oxidized phospholipid:apolipoprotein B-100 ratio with concomitant depletion of oxidized phospholipids from atherosclerotic lesions after dietary lipid-lowering. Arterioscler Thromb Vasc Biol 27, 175-181.

11. Staprans, I., Pan, X. M., Rapp, J. H., and Feingold, K. R. (2005). The role of dietary oxidized cholesterol and oxidized fatty acids in the development of atherosclerosis. Mol Nutr Food Res 49, 1075-1082.

12. Staprans, I., Rapp, J. H., Pan, X. M., Kim., K. Y., and Feingold, K. R. (1994). Oxidized lipids in the diet are a source of oxidized lipid in chylomicrons of human serum. Arterioscler Thromb 14, 1900-1905.

13. Staprans, I., Pan, X. M., Rapp, J. H., and Feingold, K. R. (2003). Oxidized cholesterol in the diet is a source of oxidized lipoproteins in human serum. J Lipid Res 44, 705-715.

14. Zilversmit, D. B. (1979). Atherogenesis: a postprandial phenomenon. Circulation 60, 473485.

15. Gorelik, S., Ligumsky, M., Kohen, R., and Kanner, J. (2008). A novel function of red wine polyphenols in humans: prevention of absorption of cytotoxic lipid peroxidation products. FASEB J 22, 41-46. 
16. Gorelik, S., Kanner, J. Schurr, D., and Kohen, R. (2013). A rational approach to prevent postprandial modification of LDL by dietary polyphenols. J Funct Food 5, 163-169.

17. Min, B., and Ahn, D. U. (2005). Mechanism of lipid peroxidation in meat and meat products-A review. Food Sci Biotechnol 14, 152-163.

18. Kanner, J., and Lapidot, T. (2001). The stomach as a bioreactor: dietary lipid peroxidation in the gastric fluid and the effects of plant-derived antioxidants. Free Rad Biol Med 31, 13881395.

19. Gorelik, S., Lapidot, T., Shaham, I., Granit, R., Ligumsky, M., Kohen, R, and Kanner, J. (2005). Lipid peroxidation and coupled vitamin oxidation in simulated and human gastric fluid inhibited by dietary polyphenols: health implications. J Agric Food Chem 53, 33973402.

20. Kanner, J. (2007). Dietary advanced lipid oxidation endproducts are a risk factor to human health. Mol Nutr Food Res 51, 1094-1101.

21. Tagliazucchi, D., Verzelloni, E., and Conte, A. (2010). Effect of dietary melanoidins on lipid peroxidation during simulated gastro-intestinal digestion: their possible role in the prevention of oxidative damage. J Agric Food Chem 58, 2513-2519.

22. Verzelloni, E., Tagliazucchi, D., and Conte, A. (2010). From balsamic to healthy: traditional balsamic vinegar melanoidins inhibit lipid peroxidation during simulated gastro-intestinal digestion of meat. Food Chem Toxicol 48, 2097-2102.

23. Sirota, R., Gorelik, S., Harris, R., M., Kohen, R, and Kanner, J. (2013). Coffee polyphenols protect human plasma from postprandial carbonyl modifications. Mol Nutr Food Res 57, 916-909.

24. Carlsen, C. U., and Skibsted, L. H. (2004). Myoglobin species with enhanced prooxidative activity is formed during mild proteolysis by pepsin. J Agric Food Chem 52, 1675-1681. 
25. Fogliano ,V., and Morales, F. J. (2011). Estimation of dietary intake of melanoidins from coffee and bread. Food Funct 2, 117-123.

26. Moreira, A. S. P., nunes, F. M., Domingues, M. R., and Coimbra, M. A., (2012). Coffee melanoidins: structure, mechanisms of formation and potential health impacts. Food Funct 3, 903-915.

27. Delgado-Andrade, C., and Morales, F. J. (2005). Unravelling the contribution of melanoidins to the antioxidant activity of coffee brew. J Agric Food Chem 53, 1403-1407.

28. Faist, V., and Erbersdobler, H. F. (2001). Metabolic transit and in vivo effects of melanoidins and precursor compounds deriving from the Maillard reaction. Ann Nutr Met $45,1-12$

29. Rufian-Henares, J. A., and Morales, F. J. (2007). Effect of in vitro enzymatic digestion on antioxidant activity of coffee melanoidins and fractions. J Agric Food Chem 55, 1001610021.

30. Reichardt, N., Gniechwitz, D., Steinhart, H., Bunzel, M., and Blaut, M. (2009).

Characterization of high molecular weight coffee fractions and their fermentation by human intestinal microbiota. Mol Nutr Food Res 53, 287-299.

31. de Marco, L. M., Fischer, S., and Henle, T. (2011). High molecular weight coffee melanoidins are inhibitors for matrix metalloproteases. J Agric Food Chem 59, 1141711423.

32. Delgado-Andrade, C., Rufian-Henares, J. A., and Morales, F. J. (2005). Assessing the antioxidant activity of melanoidins from coffee brews by different antioxidant methods. $\mathrm{J}$ Agric Food Chem 53, 7832-7836.

33. Del Pino-García, R., González-SanJosé, M. L., Rivero-Pérez, M. D., and Muñiz, P. (2012). Influence of degree of roasting on the antioxidant capacity and genoprotective effect of 
instant coffee: contribution of the melanoidin fraction. J Agric Food Chem 60, 1053010539.

34. Borrelli, R. C., Visconti, A., Mennella, C., Anese, M., and Fogliano, V. (2002). Chemical characterization and antioxidant properties of coffee melanoidins. J Agric Food Chem 50, $6527-6533$.

35. Perrone, D., Farah, A., and Donangelo, C. M. (2012). Influence of coffee roasting on the incorporation of phenolic compounds into melanoidins and their relationship with antioxidant activity of the brew. J Agric Food Chem 60, 4265-4275.

36. Gniechwitz, D., Reichardt, N., Ralph, J., Blaut, M. Steinhart, H., and Bunzel, M., (2008). Isolation and characterisation of coffee melanoidin fraction. J Food Sci Agric 88, 21532160.

37. Morales, F. J. (2005). Assessing the non-specific hydroxyl radical scavenging properties of melanoidins in a Fenton-type reaction system. Anal Chim Acta 534, 171-176.

38. Takenaka, M., Sato, N., Asakawa, H., Wen, X., Murata, M., and Homma, S. (2005). Characterization of a metal-chelating substance in coffee. Biosci Biotechnol Biochem 69 , 26-30.

39. Morales, F. J., Fernández-Fraguas, C., Jiménez-Pérez, S. (2005). Iron-binding ability of melanoidins from food and model systems. Food Chem 90, 821-827.

40. Morales, F. J., Jiménez-Pérez, S. (2004). Peroxyl radical scavenging activity of melanoidins in aqueous systems. Eur Food Res Technol 218, 515-520.

41. Daglia, M., Papetti, A., Aceti, C., Sordelli, B., Gregotti, C., and Gazzani, G. (2008). Isolation of high molecular weight components and contribution to the protective activity of coffee against lipid peroxidation in a rat liver microsome system. J Agric Food Chem 56, $11653-11660$. 
42. Goya, L., Delgado-Andrade, C., Rufián-Henares, J. A., Bravo, L., and Morales, F. J. (2007). Effect of coffee melanoidins on human heparoma hepG2 cells. Protection against oxidative stress induced by tert-butylhydroperoxide. Mol Nutr Food Res 51, 536-545.

43. Dittrich, R., Dragonas, C., Kannenkeril, D., Hoffmann, I., Mueller, A., Beckmann, M. W., and Piscetsrieder, M. (2009). A diet rich in Maillard reaction products protects LDL against copper induced oxidation ex vivo, a human intervention trial. Food Res Int 42, 1315-1322.

44. Vitaglione, P., Morisco, F., Mazzone, G., Amoruso, D. C., Ribecco, M. T., Romano, A., Fogliano, V., Caporaso, N., and D'Argenio, G. (2010). Coffee reduces liver damage in a rat model of steatohepatitis: the underlying mechanisms and the role of polyphenols and melanoidins. Hepatology, 52, 1652-1661. 


\section{FIGURE CAPTIONS}

Title of Figure 1: Changes in the levels of hydroperoxides during incubation of homogenized grilled turkey meat in simulated gastric fluid in presence of coffee melanoidins. Legend of Figure 1: The incubation at $37^{\circ} \mathrm{C}$ in absence of coffee melanoidins caused a 6.7-fold increase in the level of hydroperoxides $(\bullet)$. When coffee melanoidins were incubated at $1.5 \mathrm{mg} / \mathrm{ml}(\boldsymbol{\Delta})$ or $3.0 \mathrm{mg} / \mathrm{ml}$

(घ) in simulated gastric fluid with the meat a significant inhibition in lipid peroxidation was observed. Data are presented as mean \pm SD for three parallels for each prepared sample. Adapted with permission from Tagliazucchi et al. (2010) J Agric Food Chem 58, 2513-2519. Copyright 2010 American Chemical Society.

Title of Figure 2: Changes in secondary lipoxidation products (measured as TBA-RS) concentration during incubation of homogenized grilled turkey meat in simulated gastric fluid in presence of coffee melanoidins. Legend of Figure 2: The concentration of secondary lipoxidation products increased about 2.5-fold during incubation of meat in simulated gastric fluid ( $\mathbf{\square})$. The addition of $1.5 \mathrm{mg} / \mathrm{ml}(\square)$ or $3.0 \mathrm{mg} / \mathrm{ml}$ ( $\square$ ) of coffee melanoidins significantly inhibited the increase of TBA-RS. Data are presented as mean \pm SD for three parallels for each prepared sample. Adapted with permission from Tagliazucchi et al. (2010) J Agric Food Chem 58, 2513-2519. Copyright 2010 American Chemical Society.

Title of Figure 3: $\mathrm{Fe}^{2+}$-chelation ability of coffee melanoidins. Legend of Figure 3: $\mathrm{FeSO}_{4}(50$ $\mathrm{mg} / \mathrm{l}$ ) was incubated with food melanoidins under gastric condition. Titrations curve was created by plotting the mg of chelated $\mathrm{Fe}^{2+}$ versus the concentration of coffee melanoidins. Symbols used are: (घ) free iron and $(\square)$ chelated iron. Data are presented as mean \pm SD for three parallels for each prepared sample. Adapted with permission from Tagliazucchi et al. (2010) J Agric Food Chem 58, 2513-2519. Copyright 2010 American Chemical Society.

Title of Figure 4: Heme-binding ability of coffee melanoidins. Legend of Figure 4: Heme $(10 \mu \mathrm{M})$ was incubated with food melanoidins under gastric condition. Titrations curve was created by 
plotting $\triangle \mathrm{Abs}$ at $405 \mathrm{~nm}$ versus the concentration of coffee melanoidins. Data are presented as mean \pm SD for three parallels for each prepared sample.

Title of Figure 5. Simplified scheme showing the mechanism of $\mathrm{Fe}^{2+}$ - and heme-initiated peroxidation and the proposed mechanisms for the antioxidant activity of food melanoidins.

Legend of Figure 5: Coffee melanoidins may act at different levels. In the first step of the reaction (A) they may sequestrate $\mathrm{Fe}^{2+}$ ions and $\mathrm{HmFe}^{\mathrm{III}}$ preventing the formation of alkoxyl radical and of the protonated ferryl species $\left(\mathrm{HmFe}^{4+}-\mathrm{OH}\right)$ of heme. Coffee melanoidins may also act as radical scavenger (B) by donating hydrogen atom to the lipid peroxyl radicals (Hm, may represent the free heme moiety or the heme-containing peptides/proteins such as metmyoglobin). Reprinted with permission from Tagliazucchi et al. (2010) J Agric Food Chem 58, 2513-2519. Copyright 2010 American Chemical Society. 
Table 1. Summary of the studies focused on anti-peroxidative activity of coffee melanoidins.

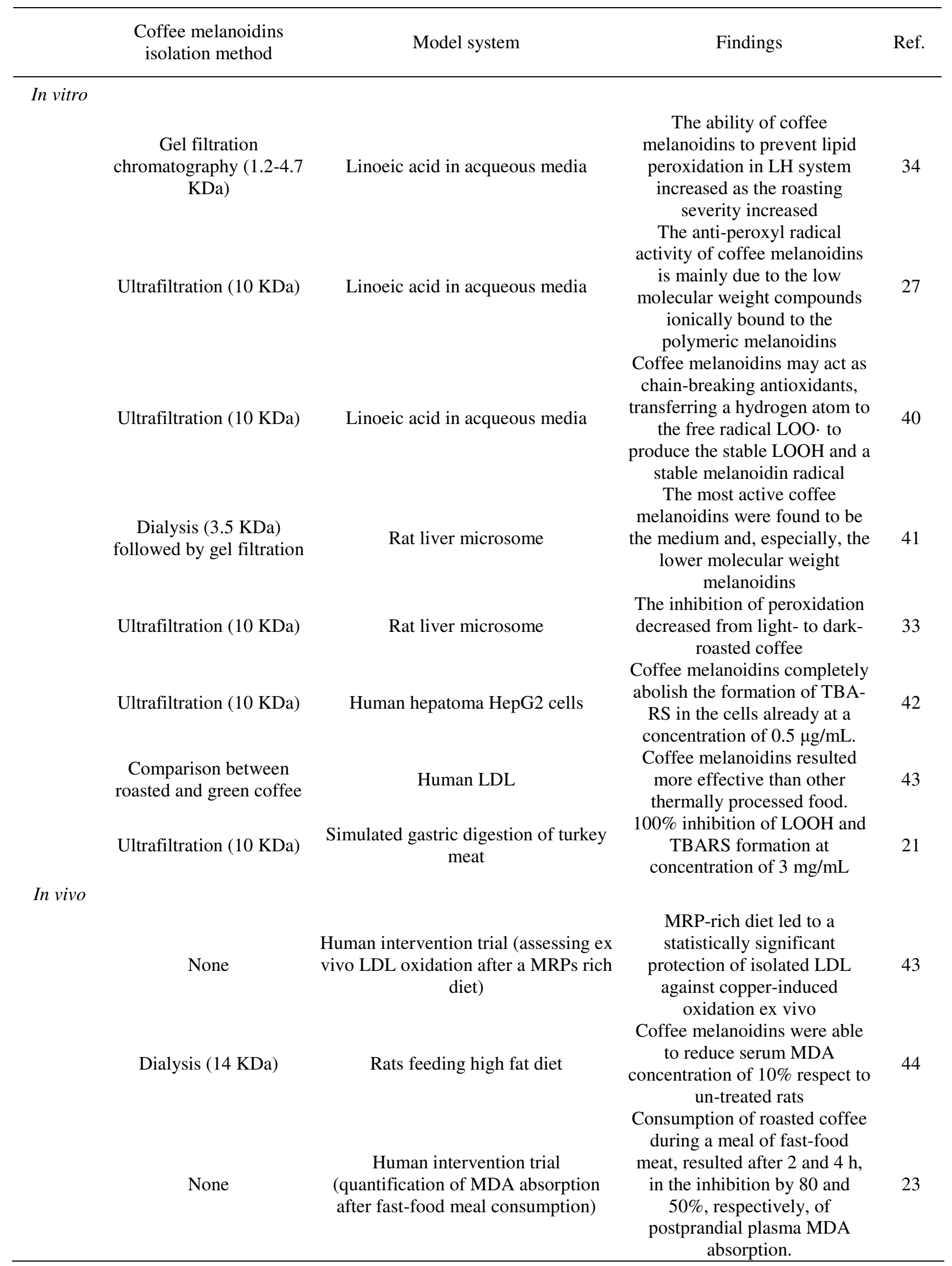


Figure 1

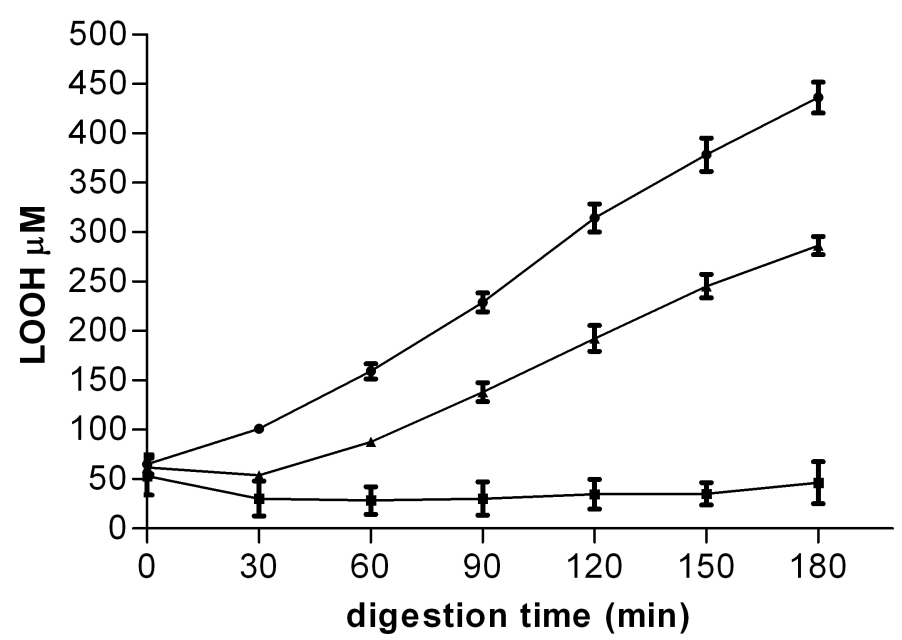


Figure 2

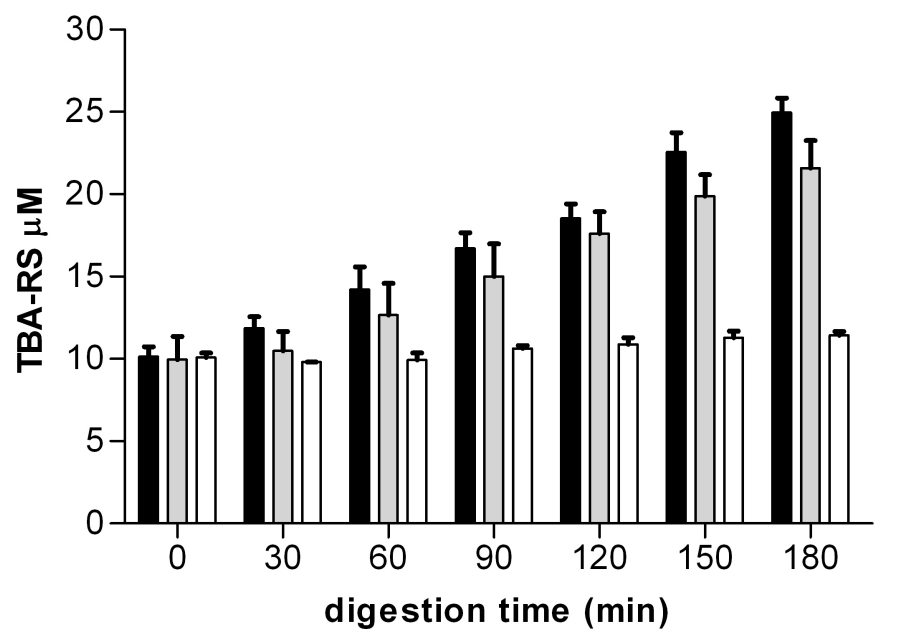


Figure 3

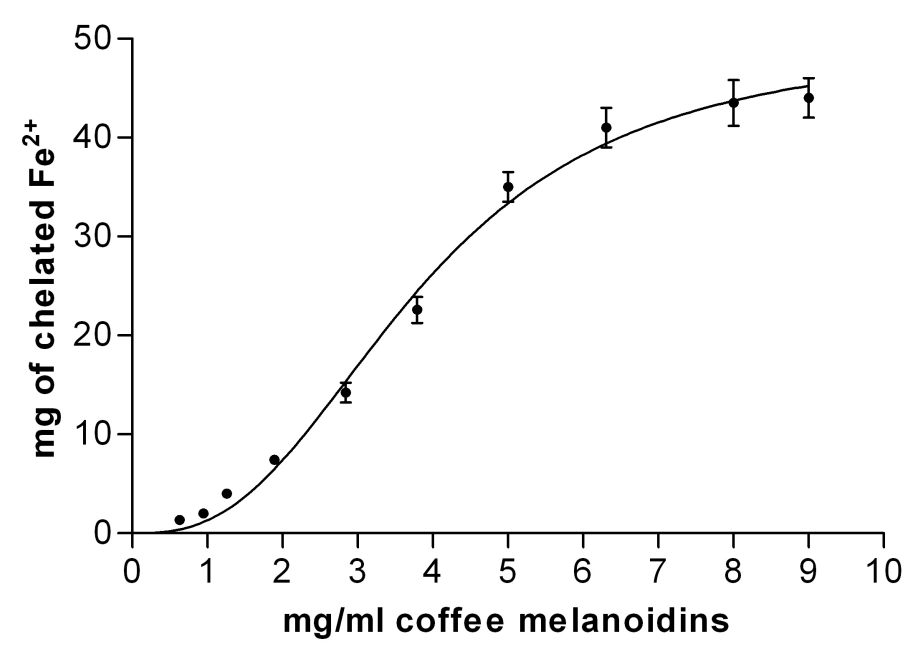


Figure 4

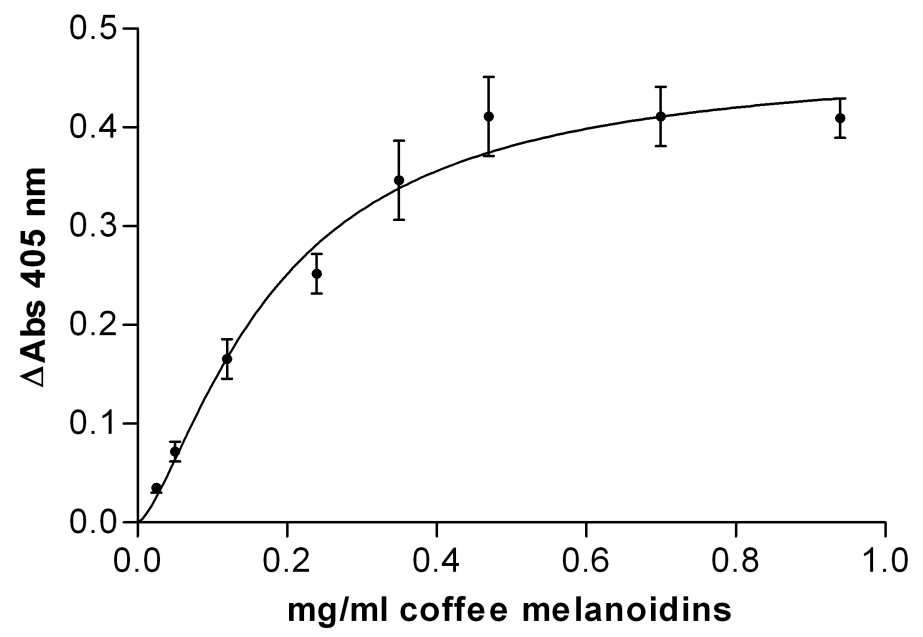


Figure 5
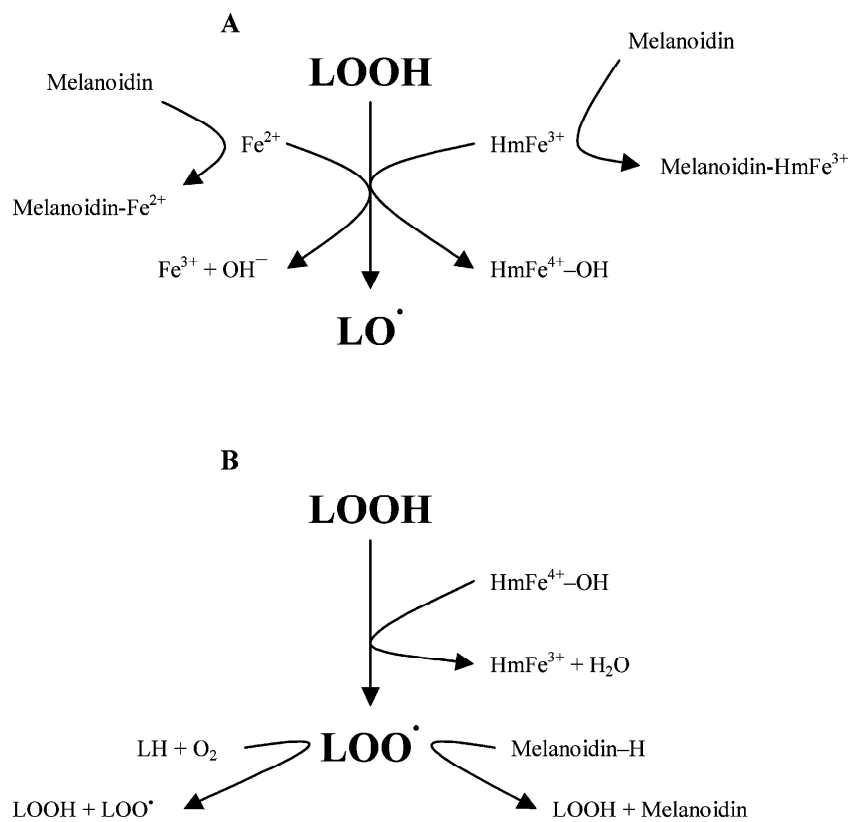